\title{
KENNY CONCEPT of TREATMENT of POLIOMYELITIS
}

\author{
By BARBARA J. BROWNLEE, B.Sc. (Rand).*
}

*Kenny Therapist in charge of the Out-patient Department, Sister Kenny Hospital for Poliomyelitis, El Monte, California.

$\mathbf{P}$ OLIOMYELITIS is an acute, infectious disease caused by a filtrable virus which attacks the anterior horn and internuncial cells and causes disorganization of the neuromuscular system. Kenny believed the disease to be systemic. This has not been proved, although the known facts tend to establish her theory.

The types of poliomyelitis are bulbar, spinal, bulbarspinal, encephalitic, abortive and nonparalytic. The Kenny method is directed towards the treatment of muscles in which are found "shortening, inco-ordination and mental alienation." and is of most benefit in the acute and early convalescent stages of the disease.

The disease is considered acute until the temperature has dropped and remained normal for 48 hours. The convalescent stage continues until maximum recovery is reached-l to 2 years after onset-and poliomyelitis is chronic when a stationary level is maintained.

The othodox concept states that the pathology is in the anterior horn cells with resulting flaccidity of the affected muscles. The normal, strong muscles pull against the weak muscles, causing a muscle imbalance which can result in deformities if untreated.

The Kenny concept (1) of the disease states that the clinical manifestations are:

Muscle spasm (shortening).

Mental alienation (pseudoparalysis).

Inco-ordination of muscle action.

Paralysis and weakness (denervation).

\section{Muscle Spasm}

Definition: A hypertonicity of muscle marked by an involuntary and persistent contraction. Because of this loss of extensibility the muscle is unatle to relax, and deformities occur if it is allowed to go untreated.

It is the earliest symptom of poliomyelitis, and is always present, even if there is no paralysis or weakness.

The muscles are hyperirritable, tender and painful. Shortening is found in paralyzed muscles, as well as in normal ones.

According to Doctor Pohl( $\left.{ }^{2}\right)$, local changes apparently take place in the muscle tissue. The hypertonicity of poliomyelitis does not always subside spontaneously. In meningitis, for example, the hypertonicity subsides as the disease regresses in the spinal cord. Doctor Pohl reasons, therefore, that nerve origin alone cannot suffice as an explanation for the shortening in poliomyelitis. Other authorities have thought that the shortening is neurogenic or vascular in origin.

There is evidence of shortening in skin, subcutaneous tissue and fascia. The skin is often tender, and becomes adherent, thick or tight, if left untreated. The skin folds are diminished, and there may be interference of normal muscle movement where there is no evidence of muscle paralysis. The pain of skin tightening is sharp, whereas that of muscle is dull. When the erectoris pilorum is involved, there is an appearance of cutis anserina. Evidence suggests that the most pain is caused by the condition of the skin fascia, or subcutaneous tissue ${ }^{(3)}$.

Mental Alienation or Pseudoparalysis

Muscles opposed to those in spasm appear. to be paralysed, flacid and toneless, but are not painful, seeming to indicate that these muscles are not directly affected. It is observed that when the shortened muscles are relaxed by treatment, there is function in the seemingly paralyzed muscles. If left untreated, permanent paralysis and atrophy may occur. The cause of alienation is not fully understood. There are thought to be possible reflex mechanisms as an explanation.

1. Reflex pain inhibition. The patient protects the painful muscle by reflexly inhibiting the action of any adjacent muscles, e.g., if the gastrocnemius is tight and painful, the anterior tibialis will make no effort to contract, as that movement would put the gastrocnemius on stretch and cause further pain.

2. Stretch paralysis. Persistent stretching of a muscle opposed to one in spasm may cause a stretch paralysis, e.g., if the gastrocnemius is tight, persistent elongation of the anterior tibialis may cause that muscle to become paralysed eventually.

3. Reciprocal innervation. This depends on the hypothesis that spasm is the result of a continuous discharge of impulses from the anterior horn cells. The theory of reciprocal inhibition states that when any muscle group is activated reflexly or voluntarily there is normally a simultaneous inhibition of the antagonist muscle group ${ }^{(1)}$.

\section{Inco-ordination}

Poliomyelitis causes disorganization of the neuromuscular system, with consequent misdirection of nerve impulses resulting in the smooth, rhythmic muscle contraction becoming inco-ordinated and ineffective, e.g.:

(a) A muscle attempts to pull from reversed origin insertion.

(b) Opposing muscles attempt to work together; the pectoralis major contracts when abduction is attempted.

(c) Unrelated muscle movements; the hamstrings contract to attempt plantar flexion.

Inco-ordination should not be confused with substitution, which is a voluntary condition.

\section{Denervation}

There is paralysis caused by injury or destruction of anterior horn cells. The condition may be temporary, with some recovery as the inflammatory process subsides. Where there is permanent destruction there is irreversible paralysis.

\section{TREATMENT}

The Kenny treatment for poliomyelitis is most effective in the acute or early convalescent stages of the diseases, After maximum relaxation and co-ordination of function have been obtained, the patient is referred to the rehabilitation service, if further treatment is necessary.

In the early stages of the disease the patient is hyperirritable, and touching or stretching lead to increased discomfort. The patient is made as comfortable as possible with the support of pillows and sandbags. Moist hot packs are ordered as soon as the diagnosis is made. The packs are made of material of at least $75 \%$ wool, which holds the heat but does not adhere to the skin. They are boiled, wrung dry and wrapped, at a temperature of 140 degrees Fahrenheit, around the part, which must be dry to prevent burning. The joints are left "free" to preserve the sensation of movement when the therapist takes the limb through a range of joint movement. Packs may also be draped over the limbs, or concentrated to an especially tight part, e.g., the chest, where breathing may be inhibited due to muscle tightness. Gentle manipulations of a stretching nature are then performed to release adherent fascia and relieve shorten- 
ing. It must be stressed that these manipulations should never cause any increase of pain or shortening.

As soon as the tenderness subsides sufficiently to allow it, muscle reeducation is begun.

The Kenny system of muscle re-education consists of: (1) Tendon stimulation.

(2) Restoration of awareness.

(3) Restoration of co-ordinated function

Tendon Stimulation. An attempt is made to stimulate the proprioceptive reflex arc. While the afferent pathway remains intact, there may be a physiological block of the efferent pathway leading to alienation or pseudoparalysis.

The patient is relaxed, and makes no attempt to help the therapist. The proprioceptive afferent pathway is stimulated by taking the joint through a small range of vibratory movement. If the efferent pathway is intact, the tendon will be seen to contract.

Restoration of Awareness. The patient is made aware of the co-ordinated, smooth conttraction of a muscle. His attention is directed towards the tendinous insertion of the muscle while the therapist takes the joint through passive movements. The afferent nerves are stimulated and consciousness established at the cerebral level.

Restoration of Co-ordination of Function. The patient attempts to help the therapist perform the movement, using only the muscles indicated. The weight of the limb is taken by the therapist, and the patient attempts to contract the muscle from the tendinous insertion indicated. No active movement is carried out if not co-ordinated, e.g.; opposing muscles must not be allowed to contract simultaneously.

Treatment is carried out on a wooden table. The complete attention of both patient and therapist is required.
As soon as is possible and without causing pain, an attempt is made to stand the patient. While in bed a footboard is placed at the foot of the bed with space between a hard mattress and the foot-board for the heels. The patient keeps his feet against the board to preserve his sense of standing. He attempts to push down against the footboard to simulate standing.

Gait training is begun when the patient has recovered sufficiently.

\section{SUMMARY}

Kenny treatment of poliomyelitis is most effective in the acute and subacute stages of the disease.

Non-paralytic poliomyelitis should be treated to prevent deformities occurring from "spasm" of muscles.

The clinical manifestations of the Kenny Concept are: Muscle "spasm"

Inco-ordination

Alienation

Denervation

While the orthodox conception is: Paralysis with flaccidity;

Muscle imbalance causing deformities. BIBLIOGRAPHY

(')POHL, JOHN F.: Kenny Concept of Infantile Paralysis and its Treatment (in collaboration with Sister Elizabeth , Kenny), Minneapolis \& Saint Paul, Minnesota, 1949.

(2) PoHL, JoHn F.: Peripheral Diseases of Poliomyelitis, J. Bone and Joint Surg., $29: 4$, Oct. 1947.

(3)KenNy, Sister ElizABETH: Physical Medicine" the Science of Dermo-Neuro-Muscular Therapy as applied to Infantile Paralysis, Minneapolis, Minnesota (published by the author), 1946.

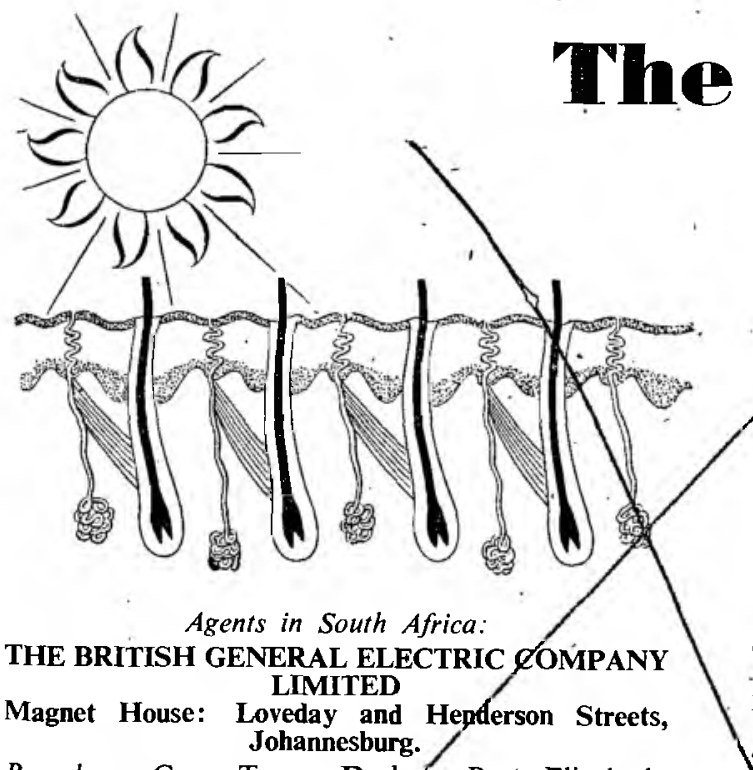

Branches: Cape Town, Durbah, Port Elizabeth, Salisbury, Bulawayo.

\section{HANOVIA}

HANOVIA LTD., SLOUGH, ENGLAND

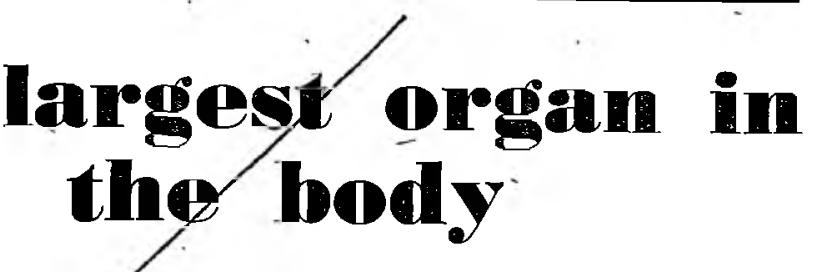

Ultra-violet irradiation is a method of administering medjeine through the skin-the largest organ in the bofá-in a form which is readily absorbed by the organism. The high-energy radiation evokes tonic reactions which stimulate all the processes of metabolism. The vitamins and anti-bodies produced in the skin and its vascular system are transmitted by the blood to the remotest cells of the organism.

This accounts for the benefits of ultra-violet irradiation in such a variety of conditions - skin diseases, blood and glandular deficiencies, sub-normal nutrition, defects of alcium metabolism, tubercular and other infections, etc. The name "Hanovia" has become synonymous with clinical equipment for ultra-violet ray treatment. Why not investigate its benefits for your own practice? Illustrated catalogue free on request. For full details contact four agents. 